\title{
Detección de anticuerpos contra el virus de distemper canino en jaguares (Panthera onca) y pumas (Puma concolor) en Madre de Dios, Perú
}

\author{
Detection of antibodies against canine distemper virus in jaguars (Panthera onca) \\ and pumas (Puma concolor) in Madre de Dios, Peru
}

\author{
José Atauje S. ${ }^{1}$, Mercy Ramírez V. ${ }^{1,3}$, Alfonso Zúñiga H.², Pedro Ospina S. ${ }^{1}$, Dennis \\ Navarro M. ${ }^{1}$, Hermelinda Rivera G. ${ }^{1}$
}

\section{Resumen}

El objetivo del estudio fue determinar la presencia de anticuerpos contra el virus de distemper canino (vDC) en jaguares (Panthera onca) y pumas (Puma concolor) de vida libre en Madre de Dios. Las 19 muestras (jaguares =13, pumas=6) fueron colectadas en la Concesión de Conservación Río Los Amigos $(n=2)$ y la Reserva Nacional Tambopata/ Parque Nacional Bahuaja Sonene $(\mathrm{n}=17)$ durante los trabajos de la World Wildlife Fund (WWF) entre 2006 y 2008. La detección de anticuerpos contra el vDC se hizo mediante la prueba de inmunofluorescencia indirecta (IFI) en una dilución de 1:50. Todos los felinos silvestres de vida libre resultaron negativos a anticuerpos contra el vDC. Los resultados indican que estos felinos no estuvieron expuestos al vDC.

Palabras clave: vDC; jaguar; puma, IFI; anticuerpos; Madre de Dios

\section{Abstract}

The objective of the study was to determine the presence of antibodies against canine distemper virus (CDv) in jaguars (Panthera onca) and pumas (Puma concolor) of free life in Madre de Dios. The 19 samples (jaguars $=13$, pumas $=6$ ) were collected the Río Los Amigos Conservation Concession $(\mathrm{n}=2)$ and the Tambopata National Reserve / Bahuaja Sonene National Park $(n=17)$ during the routine activities of the World Wildlife

\footnotetext{
${ }^{1}$ Laboratorio de Microbiología y Parasitología Veterinaria, Facultad de Medicina Veterinaria, Universidad Nacional Mayor de San Marcos, Lima, Perú

${ }^{2}$ Proyecto ÁREAS-Amazonía de la World Wildlife Fund (WWF-Perú)

${ }^{3}$ E-mail: mramirezv@unmsm.edu.pe
}

Recibido: 16 de junio de 2018

Aceptado para publicación: 10 de noviembre de 2018 
Fund (WWF) in 2006-2008. The detection of antibodies against the CDv was carried out by the indirect immunofluorescence test (IFAT) in a dilution of 1:50. All free-living wild cats were negative for antibodies to CDv. The results indicate that these cats were not exposed to CDv.

Key words: CDv; jaguar; puma; IFAT; antibodies; Madre de Dios

\section{INTRODUCCIÓN}

En las últimas décadas se han observado numerosos brotes de enfermedades infecciosas en poblaciones de animales silvestres causadas por patógenos de animales domésticos ocasionando una reducción en su población (Daszak et al., 2000; Tompkins et al., 2015). Entre estos se encuentran el virus de distemper en focas, parvovirosis en leones, micosis en anfibios y tuberculosis en mustélidos (Daszak y Cunningham, 2002). El virus de distemper canino (vDC) es el agente viral con mayor impacto en la salud de las poblaciones de carnívoros silvestres, pudiendo ocasionar una alta tasa de mortalidad en poblaciones inmunológicamente no expuestas (Quigley et al., 2010); entre estos, el brote de distemper canino (DC) en perros silvestres (Lycaon pictus) en Tanzania que ocasionó la muerte de 49 cánidos silvestres en dos meses (van de Bildt et al., 2002) y el reconocido brote en el Parque Nacional de Serengeti en Tanzania en 1994, donde murieron 23 leones (Panhera leo) (Roelke-Parer et al., 1996).

Existen reportes de la exposición de felinos silvestres al virus de distemper canino (vDC) sin casos de mortalidad en Brasil, donde el $31.5 \%$ de jaguares (Panthera onca) y $11.3 \%$ de pumas (Puma concolor) tuvieron anticuerpos contra el vDC (Nava et al., 2008). Así mismo, Fiorello et al. (2007) reportaron anticuerpos contra el vDC en ocelotes (Leopardus pardalis) y zorros grises de las pampas (Lycalopex gymnocercus). En otro estudio utilizando la prueba de RT-PCR se detectaron 3 de 5 tigres de Amur (Panthera tigris altaica) positivos al vDC en China y Rusia (Seimon et al., 2013). Así mismo, Sulikhan et al. (2018) encontraron a un leopardo de Amur (Panthera pardus orientalis) en China expuesto al vDC por medio de RT-PCR. En Costa Rica se detectaron $2 \%$ de los felinos silvestres positivos al vDC en muestras de heces usando la secuencia del gen de la fosfoproteína $\mathrm{P}$ del virus (Avendaño et al., 2016).

Entre las causas disruptivas de la ecología de una región y facilitadoras de enfermedades infecciosas emergentes en especies silvestres se encuentran las alteraciones del hábitat, la sobreexplotación, las interacciones con especies alóctonas depredadoras o competidoras y las migraciones a nuevos hábitat o regiones. Así mismo, las interacciones con nuevos huéspedes vectores (Medina y Vogel, 2010) como el perro doméstico (Canis lupus familiaris), especie reconocida por su capacidad para propagar el vDC (Almberg et al., 2010; Viana et al., 2015), causando mortalidad en carnívoros silvestres, tanto de vida libre como en cautiverio (van de Bildt et al., 2002).

Evaluar el riesgo de la diseminación de estas enfermedades en poblaciones de animales de vida silvestre en el país es complicado por la falta de un estudio base que permita determinar si las especies de felinos silvestres de vida libre están expuestos a un patógeno como el vDC y, luego, evaluar si su presencia constituye una potencial amenaza para estas poblaciones. Por esta razón, el objetivo del presente trabajo fue determinar si las muestras de pumas (P. concolor) y jaguares $(P$. onca) obtenidas como parte del trabajo de campo de la World Wilde Fund, WWF-Perú, estaban expuestas al vDC. 


\section{Materiales y Métodos}

Se utilizaron 19 muestras de suero de felinos silvestres adultos; 13 jaguares (Panthera onca) y 6 pumas (Puma concolor), provenientes de la Concesión de Conservación Río Los Amigos (Los Amigos) $(n=2)$ y la Reserva Nacional Tambopata/Parque Nacional Buhuaja Sonene (RNTMB/ PNBS) de Madre de Dios (n=17) (Figura 1). Cinco $P$. concolor fueron machos y una fue hembra. En el caso de $P$. onca, ocho fueron machos y cinco fueron hembras.

Las muestras fueron recolectadas durante las labores de campo del proyecto Áreas-Amazonía de la WWF-Perú entre los años 2006-2008, utilizando trampas lazo para la captura de los felinos (Figura 2). Los sueros estaban en congelación en el banco de sueros del Laboratorio de Virología de la Facultad de Medicina Veterinaria de la Universidad Nacional Mayor de San Marcos (SVLMP, FMV-UNMSM) en Lima, Perú.

Para la determinación de anticuerpos contra el vDC se utilizó la prueba de inmunofluorescencia indirecta (IFI) empleando el kit Canine Distemper Virus (CDV) FITC Conjugate Mab y un suero de origen canino positivo a anticuerpos contra el vDC (VMRD, EEUU), utilizando el protocolo disponible de la SV-LMP, FMV-UNMSM.

\section{Resultados y Discusión}

Las 19 muestras resultaron seronegativas contra el vDC, indicando la ausencia de contacto directo con animales domésticos. Resultados similares fue reportado en muestras de sueros de margays (Leopardus wiedii) rescatados de una zona de reserva en Guatemala y que no habían tenido contacto con animales domésticos; sin embargo, las muestras de suero de gatos domésticos fueron positivos a anticuerpos contra el vDC ya que estos tenían contacto con perros de la cercana ciudad de Petén (Lickey et al.,
2005). Así mismo, muestras de 84 felinos silvestres mantenidos en cautiverio en Brasil resultaron negativas a anticuerpos neutralizantes contra el vDC, concluyéndose que el virus no circulaba en las poblaciones de felinos silvestres de esa área (Schmidt, 2006).

Una de las razones de la ausencia de anticuerpos contra el vDC en los 19 felinos estudiados podría ser los $40 \mathrm{~km}$ de distancia que existe entre la zona de estudio (RNTMB/ PNBS) y el asentamiento poblado más cercano, que es la comunidad nativa de Infierni1lo, lo que haría difícil el contacto entre los perros o gatos domésticos infectados con el vDC y la población susceptible de felinos silvestres. Cabe mencionar que no existe población humana asentada en la zona del estudio y que los guarda parques controlan la entrada de personas a la RNTMB (A. Zuñiga, Comunicación personal).

En el caso de la Concesión de Conservación Río los Amigos, el centro poblado más cercano al área donde de muestreo es la ciudad Boca-Amigos, ubicada a $4 \mathrm{~km}$, seguida de San Juan a $12 \mathrm{~km}$ y Shiringayos a $20 \mathrm{~km}$, todos en el eje del río Madre de Dios. También existen pequeños campamentos mineros y muchos de ellos tienen mascotas. Los animales infectados, para trasmitir el vDC, tendrían que estar en altas densidades para mantener el patógeno circulando, caso que no ocurre en las comunidades porque al haber pocas mascotas, el ciclo de transmisión se completaría relativamente rápido a todos los animales del lugar haciendo que la infección se vuelva autolimitante (A. Zuñiga, comunicación personal). Otra de las razones del resultado negativo a la exposición al vDC es la baja densidad de felinos silvestres en las áreas muestreadas y el comportamiento solitario de estos animales que hace difícil la transmisión del vDC (Tobler et al., 2013).

La prueba IFI detecta anticuerpos producto de una exposición de virus de campo o de vacuna utilizando un anticuerpo policlonal anti-IgG felino marcado con FITC, debido a- 


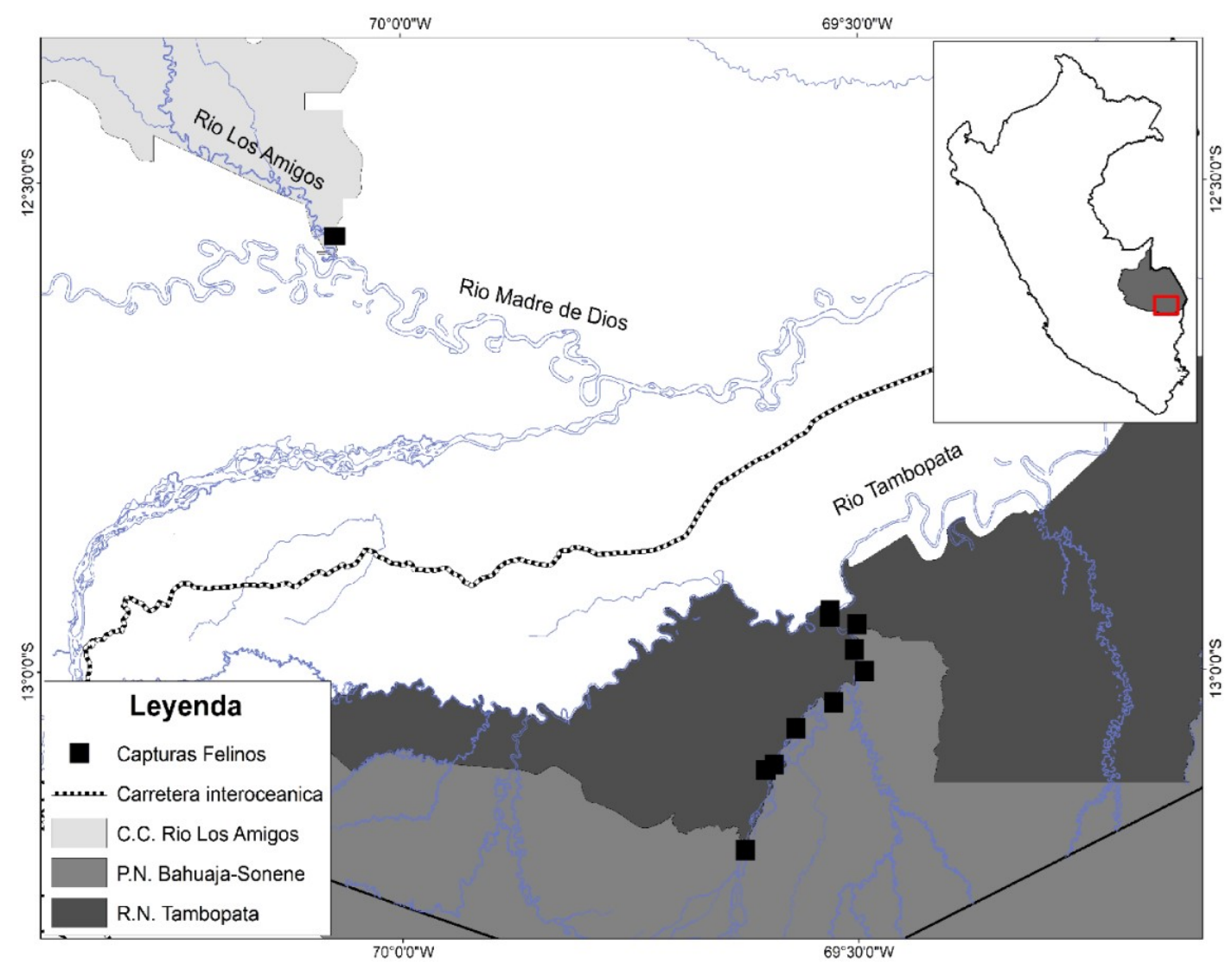

Figura 1. Mapa de ubicación de la Concesión de Conservación Río Los Amigos (Los Amigos) y la Reserva Nacional Tambopata/Parque Nacional Buhuaja Sonene (RNTMB/PNBS) de Madre de Dios. Las áreas sombreadas son áreas protegidas. La línea punteada oscura muestra la carretera interoceánica

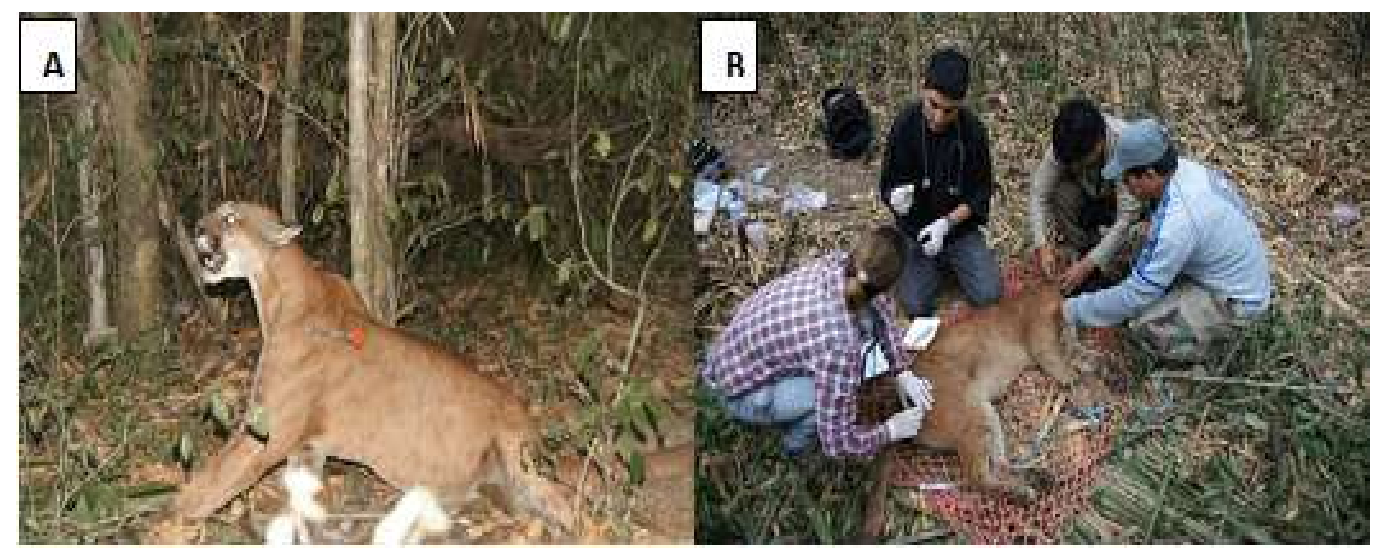

Figura 2. Labores de campo del proyecto Áreas-Amazonía de la WWF-Perú entre los años 2006-2008. A: Sedación de un puma (P. concolor) mediante cerbatana. B. Toma de muestras y colocación de radio collares en (P. concolor). Foto: WWF-Perú / Jeff Sikkich 
la cercanía filogenética entre felinos domésticos (Felis catus) y silvestres (Montague et al., 2014) permitiendo identificar el isotipo deseado (MacLachlan y Dubovi, 2011). La prueba de IFI es considerada por la Unión Internacional para la Conservación de la Naturaleza (UICN) y la Organización Mundial de Sanidad Animal (OIE) como de baja utilidad, pero de alta sensibilidad para la detección de anticuerpos contra agentes virales en fauna silvestre (UICN, 2016). Esto se encuentra respaldado por resultados positivos a anticuerpos contra Calicivius felino en felinos silvestres de un zoocriadero de Lima utilizando el mismo anti-IgG felino, lo cual corroboraría la unión especifica del policlonal con el anticuerpo de IgG de pumas y jaguares utilizados en el presente estudio (M. Ramírez, comunicación personal).

La ausencia de anticuerpos contra el vDC en pumas y jaguares de este estudio no garantiza que la población felina no esté expuesta a este virus. No obstante, las políticas de seguridad que son implementadas por el Servicio Nacional de Áreas protegidas por el Estado (SERNAMP), las concesiones de conservación y las áreas de concesiones forestales en Madre de Dios están bien llevadas, lo que permite minimizar los contactos entre los animales domésticos y la fauna silvestre (Tobler et al., 2018). Sin embargo es necesario seguir monitoreando a la población de felinos silvestres y su exposición a diferentes enfermedades infecciosas, así como establecer el rol de los carnívoros domésticos en la ecología de estos agentes infecciosos (Avendaño et al., 2016).

Este es el primer trabajo sobre el vDC en jaguares (Panthera onca) y pumas (Puma concolor) de vida libre en el Perú.

\section{Conclusiones}

No se encontraron anticuerpos contra el virus del distemper canino en las 19 muestras de suero de pumas (Puma concolor) y- jaguares (Panthera onca) colectadas en el periodo 2006-2008 en la Concesión de Conservación Río Los Amigos (Los Amigos) y la Reserva Nacional Tambopata/Parque Nacional Buhuaja Sonene (RNTMB/PNBS).

\section{Agradecimientos}

Al Dr. Alfonso Zuñiga y a la Dra. Paloma Alcázar, miembros de la WWF, por brindar las muestras y la información de felinos silvestres de la RNTMB/PNBS y de Los Amigos. El estudio fue financiado por el Vicerrectorado de Investigación y Posgrado de la Universidad Nacional Mayor de San Marcos (VRIP-UNMSM) (RR 06369-R-17 con código: A17080424b).

\section{Literatura Citada}

1. Almberg ES, Cross PC, Smith DW. 2010. Persistence of canine distemper virus in the Greater Yellowstone Ecosystem's carnivore community. Ecol Appl 20: 2058-2074. doi: 10.1890/091225.1

2. Avendaño $R$, Barrueta $F$, SotoFournier $S$, Chavarría M, Monge $O$, Gutiérrez-Espeleta GA, Chaves A. 2016. Canine distemper virus in wild felids of Costa Rica. J Wildlife Dis 52: 373-377. doi: 10.7589/2015-02-041

3. Daszak P, Cunningham AA, Hyatt AD. 2000. Emerging infectious diseases of wildlife-threats to biodiversity and human health. Science 287: 443-449. doi: 10.1126/science.287.5452.443

4. Daszak P, Cunningham AA. 2002. Emerging infectious diseases. A key role for conservation medicine. In: Aguirre AA, Ostfeld RS, Tabor GM, Pearl MC (eds). Conservation Medicine. New York: Oxford University Press. p 40-61.

5. Fiorello CV, Noss AJ, Deem SL, Maffei L, Dubovi EJ. 2007. Serosurvey of small carnivores in the Bolivian Chaco. J Wildlife Dis 43: 551557. doi: 10.7589/0090-3558-43.3.551 
6. Lickey ALA, Kennedy M, Patton S, Ramsay EC. 2005. Serologic survey of domestic felids in the Petén region of Guatemala. J Zoo Wildlife Med 36: 121-123. doi: 10.1638/03-059

7. MacLachlan NJ, Dubovi EJ. 2011. Fenner's veterinary virology. $4^{\circ}$ ed. California: Elsevier. $534 \mathrm{p}$.

8. Medina-Vogel G 2010. Ecología de enfermedades infecciosas emergentes y conservación de especies silvestres. Arch Med Vet 42: 11-24. doi: 10.4067/ S0301-732X2010000100003

9. Montague MJ, Li G, Gandolfi B, Khan $R$, Aken BL, Searle SM, Minx P, et al. 2014. Comparative analysis of the domestic cat genome reveals genetic signatures underlying feline biology and domestication. P Natl Acad Sci USA 111: 17230-17235. doi: 10.1073/ pnas. 1410083111

10. Nava AFD, Cullen JL, Sana DA, Nardi MS, Ramos Filho JD, Lima TF, Abreu KC, et al. 2008. First evidence of canine distemper in Brazilian freeranging felids. Ecohealth 5: 513-518. doi: 10.1007/s10393-008-0207-8

11. Quigley KS, Evermann JF, Leathers CW, Armstrong DL, Goodrich J, Duncan NM, Miquelle DG. 2010. Morbillivirus infection in a wild Siberian tiger in the Russian Far East. J Wildlife Dis 46: 1252-1256. doi: 10.7589/00903558-46.4.1252

12. Roelke-Parker ME, Munson L, Packer C, Kock R, Cleaveland S, Carpenter M, Obrien SJ, et al. 1996. A canine distemper virus epidemic in Serengeti lions (Panthera leo). Nature 379: 441-445. doi: 10.1038/379441a0

13. Schmidt HTL. 2006. Anticuerpos neutralizantes contra los virus de distemper y parainfluenza canino en caninos y felinos silvestres en cautiverio. Tesis de Médico Veterinario. Puerto Alegre, Brasil: Univ. Federal de Rio Grande del Sur. $72 \mathrm{p}$.

14. Seimon TA, Miquelle DG, Chang TY, Newton AL, Korotkova I, Ivanchuk G, et al. 2013. Canine distemper virus: an emerging disease in wild endangered
Amur tigers (Panthera tigris altaica). Mol Biol 4: 410-413. doi: 10.1128/ mBio.00410-13

15. Sulikhan NS, Gilbert M, Blidchenko EY, Naidenko SV, Ivanchuk GV, Gorpenchenko TY, Alshinetskiy MV, et al. 2018. Canine distemper virus in a wild far eastern leopard (Panthera pardus orientalis). J Wildlife Dis 54: 170-174. doi: 10.7589/2017-03-065

16. Tobler M, Carrillo-Percastegui SE, Zúñiga HA, Powell, George VN. 2013. High jaguar densities and large population sizes in the core habitat of the southwestern Amazon. Biol Conserv 159: 375-381. doi: 10.1016/j.biocon.2012.12.012

17. Tobler M, García R, CarrilloPercastegui S, Ponce G, Polisar J, Zuñiga HA, Goldstein I. 2018. Do responsibly managed logging concessions adequately protect jaguars and other large and medium- sized mammals? Two case studies from Guatemala and Peru. Biol Conserv 220: 245-253. doi: 10.1016/j.biocon.2018.02.015.

18. Tompkins DM, Carver S, Jones ME, Krkošek M, Skerratt LF. 2015. Emerging infectious diseases of wildlife: a critical perspective. Trends Parasitol 31: 149-159. doi: 10.1016/j.pt.2015.01.007

19. [UICN] Unión Internacional para Conservación de la Naturaleza. 2016. Manual de procedimientos para el análisis del riesgo de enfermedad en fauna silvestre. París: UICN. 172 p.

20. van de Bildt MW, Kuiken T, Visee AM, Lema S, Fitzjohn TR, Osterhaus AD. 2002. Distemper outbreak and its effect on African wild dog conservation. Emerg Infect Dis 8: 212-213. doi: 10.3201/ eid0802.010314

21. Viana M, Cleaveland S, Matthiopoulos $J$, Halliday J, Packer C, Craft ME, Hampson K, et al. 2015. Dynamics of a morbillivirus at the domestic-wildlife interface: canine distemper virus in domestic dogs and lions. P Natl Acad Sci USA 112: 1464-1469. doi: 10.1073/ pnas. 1411623112 